Classification

Physics Abstracts

$64.70 \mathrm{~J}-68.10 \mathrm{C}-82.70$

\title{
Critical behaviour in microemulsions
}

\author{
A. M. Cazabat, D. Langevin, J. Meunier and A. Pouchelon \\ Laboratoire de Spectroscopie Hertzienne de l'E.N.S., \\ 24, rue Lhomond, 75231 Paris Cedex 05, France
}

(Reçu le 10 septembre 1981, révisé le 18 novembre, accepté le 14 décembre 1981)

\begin{abstract}
Résumé. - Nous avons observé un comportement critique dans les microémulsions formant partie de systèmes polyphasiques. Nous avons étudié les propriétés volumiques et interfaciales de ces systèmes à l'aide de techniques optiques. Le comportement critique a été trouvé plus marqué sur les propriétés interfaciales. Les expériences sont en bon accord avec les théories de couplage de modes.
\end{abstract}

\begin{abstract}
Critical behaviour was observed in multiphase microemulsion systems. Interfacial and bulk properties were both studied through optical techniques. The critical behaviour was found to be more pronounced on interfacial properties. The experiments are in good agreement with modemode coupling theories.
\end{abstract}

1. Introduction. - Microemulsions [1] are transparent dispersions of two immiscible fluids like water and oil, made with the help of amphiphilic molecules. The most common idea relative to the structure of the dispersion is that of small droplets of water (or oil) surrounded by a film of amphiphilic molecules in a continuous medium formed by the oil (or water). Microemulsions have numerous practical applications : photochemistry, drugs delivery, oil recovery, ...

Amphiphilic compounds can lower the oil-water interfacial tension $\gamma$, initially of $35-50 \mathrm{dyn} . / \mathrm{cm}$, down to values as low as $10^{-3} \mathrm{dyn} . / \mathrm{cm}$. It has been suggested that, when $\gamma$ is very low, the increase of free energy due to creation of large interfacial area may be balanced by an entropy increase due to dispersion, thus explaining the stability of these systems [2]. An important lowering of interfacial tension is expected to be achieved when the amphiphile molecules affinities for oil and water are similar, thus leading to large amounts of adsorbed molecules at the interface. In practice this condition is usually not fulfilled. However, it is possible to adjust the affinities through modifying either oil or water by adding other compounds like alcohols or salts, or by varying the temperature.

A four component system oil-water-amphiphile-alcohol has a complex phase diagram. At a fixed temperature, microemulsions single phase domains can be found; microemulsions can also coexist with excess aqueous or organic phases in two phases domains, or with both excess aqueous and organic phases in three phases domains. The interfacial tensions between the microemulsions phases and the excess phases are extremely small, of the order of $10^{-3} \mathrm{dyn} . / \mathrm{cm}$. It has been suggested that the origin of these low interfacial tensions no longer lies on the structure of 
the interfacial layer as in the above mentioned oil-water interfaces, but rather on the structure of the microemulsion phase itself, and is associated to the vicinity of critical consolute points [3].

The near-critical microemulsion phases show a turbidity enhancement that can be confused with an increase of droplets size. At the same time, angular variations of the scattered intensity and diffusion coefficient can be attributed to apparent long range forces between droplets. We present here a more correct treatment in terms of critical concentration fluctuations. We report also measurements of interfacial tensions and interfacial thicknesses that support this interpretation.

2. Experimental results. - We have studied as model systems, mixtures of water + sodium chloride (46.8 wt. \%), toluene (47.2 wt. \%), sodium dodecyl sulfate (SDS, 2 wt. \%) and butanol ( 4 wt. $\%$ ). The temperature of the experiments is $20^{\circ} \pm 0.1{ }^{\circ} \mathrm{C}$. We have studied the phase behaviour obtained by varying the amount of sodium chloride in the mixture in order to decrease the solubility of the amphiphile in water. At low salinities, i.e. percentages of sodium chloride in water below $S_{1}=5.35 \mathrm{wt} \%$, SDS is more soluble in water : the mixture separates into an oil in water microemulsion in equilibrium with an organic phase. At larger salinities $S>S_{2}=7.38 \mathrm{wt} . \%$, SDS is more soluble in the oil : we obtain water in oil microemulsions in equilibrium with aqueous phases. At intermediate salinities $S_{1}<S<S_{2}$ the microemulsion coexists with both aqueous and organic phases.

We have studied the interfaces between the different phases in equilibrium : interfacial tensions $\gamma$ were measured with surface light scattering techniques [4], interfacial thicknesses $L$ were deduced from reflectivity measurements [5]. The densities of the phases in contact have been measured with a picnometer. We have also studied the microemulsion phases with bulk elastic and quasielastic light scattering techniques. These data can be interpreted by assuming that the light is scattered by the droplets submitted to Brownian motion. The analysis leads to droplets radii, virial coefficients related to interaction forces, and when the range of forces is large the pair distribution function [6]. Let us note however that this interpretation can be made only if samples of different droplets concentrations are studied : determination of radii for instance involves extrapolations to zero droplets volume fraction. For this purpose we have worked out a dilution procedure of the microemulsions in the two phase domains $S<S_{1}$ and $S>S_{2}$ [7]. In the three phase domain $S_{1}<S<S_{2}$, all our attempts failed. We believe that this is because the droplets structure no longer exists. Indeed the microemulsion phase must invert from an oil in water for $S<S_{1}$ to a water in oil structure for $S>S_{2}$. Among different possibilities, bicontinuous structures were proposed (sponge-like structures) [8]. The microemulsion phase could also be a true molecular solution among the constituents.

In the two phase domains, bulk light scattering measurements were performed on the microemulsion phases and on their dilutions. Far from the boundaries $S_{1}$ and $S_{2}$ the droplets tend to behave like hard spheres (table I). When approaching $S_{1}$ or $S_{2}$ the droplets sizes increase and attractive interactions appear and increase. These attractive interactions can be attributed to Van der Waals forces and are unusually strong in microemulsions because droplets can interpenetrate [9].

The increase of interaction forces is associated to strong modifications of the electrical properties. Close to $S_{2}$ a percolation phenomenon is observed when the volume fraction of the droplets is varied at fixed salinity (Fig. 1). Above a volume fraction $\varphi^{*}$, the conductivity increases steeply by several orders of magnitude. The percolation threshold corresponds to the volume fraction $\varphi^{*}$ above which transient interconnections between droplets are established on a macroscopic scale. At larger salinities, the percolation phenomenon disappears, probably because in hard-sphere like dispersions, the contact times between droplets are too short. Let us also point out that, for each salinity, the volume fraction of the initial microemulsions seems to correspond to the percolation threshold $\varphi^{*}$. 
Table I. - Examples of microemulsions characteristics for different salinities : $S_{1}=5.4$ and $S_{2}=7.4$ are the limits of two phases domains. The radius $R$ and virial coefficient $B$ are related to osmotic compressibility through $\frac{\partial \pi}{\partial \varphi}=\frac{3 k_{\mathrm{B}} T}{4 \pi R^{3}}(1+B \varphi), \varphi$ being the droplets volume fraction. The hydrodynamic radius $R_{\mathrm{H}}$ and virial coefficient $\alpha$ are related to diffusion coefficient through

$$
D=\frac{k_{\mathrm{B}} T}{6 \pi \eta_{0} R_{\mathrm{H}}}(1+\alpha \varphi)
$$

$\eta_{0}$ being the viscosity of the continuous phase. For hard spheres $B=8, \alpha=1.5$. The difference between $R$ and $R_{\mathrm{H}}$ close to $S_{1}$ can be attributed to polydispersity and non spherical shape of droplets [5].

\begin{tabular}{|c|c|c|c|c|}
\hline$S$ & $R$ & $R_{\mathrm{H}}$ & $B$ & $\alpha$ \\
\hline - & - & - & - & - \\
\hline 3.5 wt. $\%$ & $85 \AA$ & $95 \AA$ & 5 & -1 \\
\hline 5 & 130 & 190 & 3 & -5 \\
\hline 5.2 & 155 & 240 & 0 & -6 \\
\hline 8 & 180 & 200 & 2 & -7 \\
\hline 10 & 125 & 150 & 6 & -2 \\
\hline
\end{tabular}

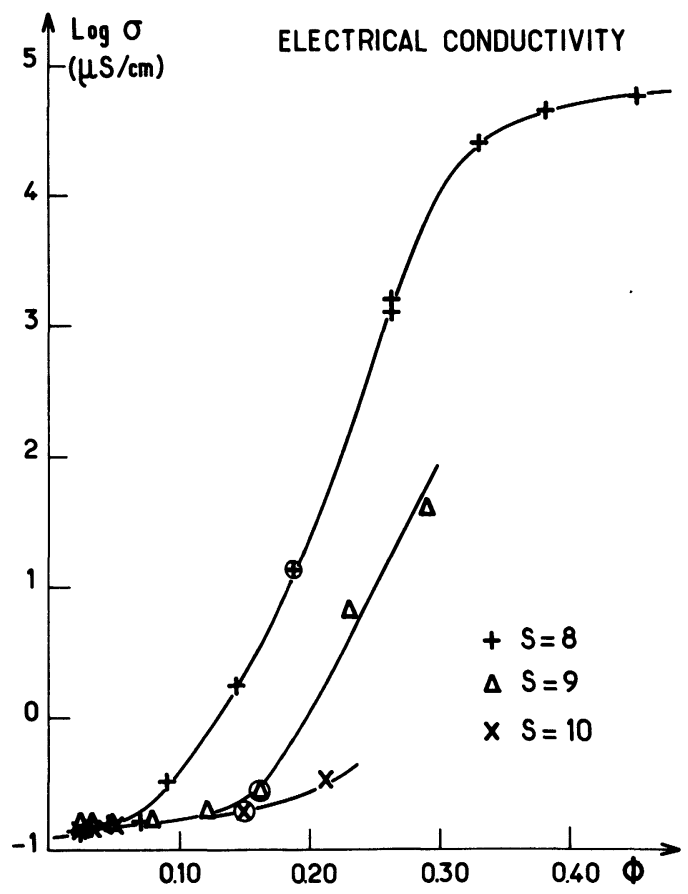

Fig. 1. - Microemulsion electrical conductivity versus droplets volume fraction at different salinities $S$. Circles correspond to the initial microemulsions as obtained from phase separation.

The increase of interaction forces close to $S_{2}$ is also associated to the onset of intensity and diffusion coefficient variations versus scattering angle. This can be interpreted by assuming that attractive interactions are long ranged. A similar behaviour was also observed for other micro- 
emulsions close to a percolation threshold when strong attractive forces were present [10]. However, the long range character of interactions can hardly be understood, since they originate on Van der Waals forces. As pointed out before, the light scattering characteristics can also be the manifestation of the vicinity of a critical point. We then tried to interpret the data in terms of critical concentration fluctuations. The scattered intensity data were fitted with Ornstein-Zernike law : $I(q) \sim\left(1+q^{2} \xi^{2}\right)^{-1}$ where $q$ is the scattering wave vector, and the diffusion coefficient data with Kawasaki formula $D(q)=D_{0} K(q \xi) / q^{2} \xi^{2}$ with

$$
K(x)=\frac{3}{4}\left|x^{2}+1+\left(x^{3}-\frac{1}{x}\right) \operatorname{Arctg} x\right| ; \quad D_{0}=k_{\mathrm{B}} T / 6 \pi \eta \xi,
$$

$\eta$ being the viscosity of the microemulsion [11]. Three independent determinations of the correlation length $\xi$ are obtained in this way. They are reported on figure 2 and table II. The agreement

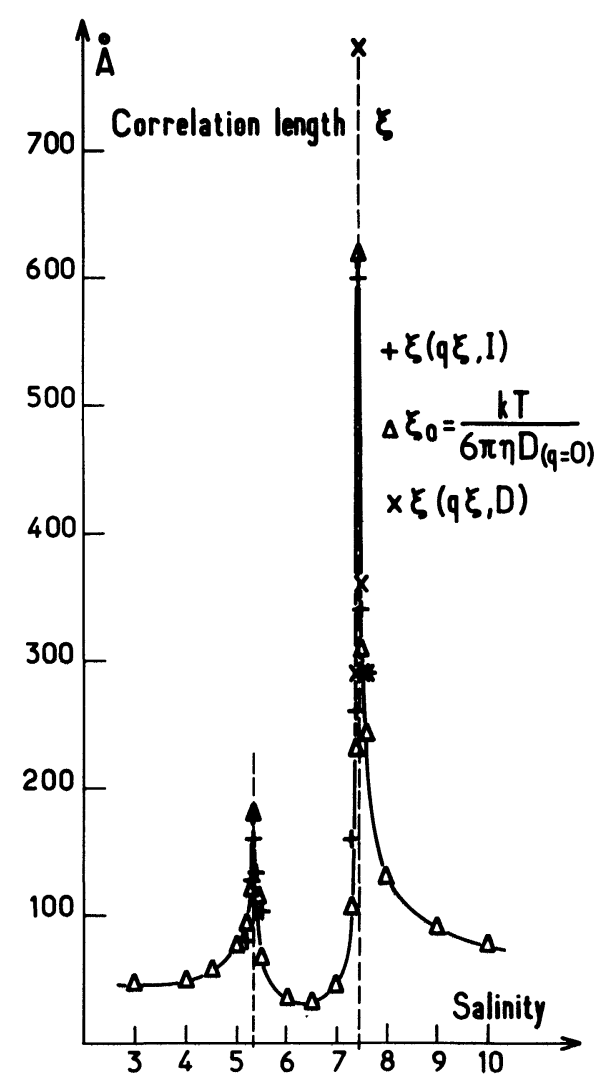

Fig. 2. - Correlation lengths in the microemulsion phases versus salinity. Vertical dotted lines show the limits between two phases and three phases domains; three different determinations of $\xi$ are obtained : $+:$ from Ornstein-Zernike formula; $\times$ : from Kawasaki formula; $\Delta:$ from $D(q=0)$ as indicated in the text.

is good close to the salinity $S_{2}$. The fact that near-critical microemulsions have droplets volume fractions close to percolation threshold values has still to be understood. Close to $S_{1}$, the agreement is less satisfactory : $I$ is $q$ dependent whereas $D$ is not. The description in terms of individual droplets is still more appropriated at least for $S<S_{1}$ (two phase domain) [7]. 
Table II. - Correlation lengths $\xi$ in the microemulsion phases as deduced from :

$$
\xi_{0}=\frac{k T}{6 \pi \eta D(q=0)},
$$

$\xi(I)$ : Ornstein-Zernike law, $\xi(D):$ Kawasaki formula.

\begin{tabular}{|c|c|c|c|}
\hline$S$ & $\xi_{0} \AA$ & $\xi(I) \AA$ & $\xi(D) \AA$ \\
\hline- & - & - & - \\
\hline 5.2 & 100 & 90 & $\lesssim 100\left(^{*}\right)$ \\
\hline 5.3 & 120 & 125 & - \\
\hline 5.4 & 190 & 170 & - \\
\hline 5.5 & 110 & 130 & - \\
\hline 5.6 & 80 & 100 & - \\
\hline 7.2 & 110 & 150 & - \\
\hline 7.3 & 220 & 250 & 280 \\
\hline 7.4 & 610 & 600 & 750 \\
\hline 7.5 & 300 & 320 & 350 \\
\hline 7.6 & 230 & 280 & 280 \\
\hline
\end{tabular}

$(*)$ No measurable angular anisotropy.

Evidences of the vicinity of a critical point also appear on other physical properties :

- When the salinity $S$ approaches $S_{2}$, the top organic phase in the three phase domain changes continuously into a turbid phase before disappearing. Similarly close to $S_{1}$ the bottom aqueous phase changes continuously into a turbid phase.

- The interfacial tension between middle phase microemulsions and the disappearing phases are very low, below $10^{-3} \mathrm{dyn} . / \mathrm{cm}$; the corresponding interfacial thickness are very large, up to $1000 \AA$. The first observation suggests that $S_{1}$ and $S_{2}$ are close to two critical end points in the phase diagram [12]. One might expect the physical properties to obey critical scaling laws in the vicinity of these points.

3. Discussion. - Let us consider a multicomponent system in a two (or more) phase region at constant temperature and pressure. From the theory of critical phenomena [13-15] the difference between the concentrations of a component $i$ in the two phases must approach zero at the critical (plait or end) point according to :

$$
\Delta C_{i}=\Delta C_{i}^{0} \varepsilon^{\beta}
$$

where $\varepsilon=\left|\left(\mu_{i}-\mu_{i}^{\mathrm{C}}\right) / \mu_{i}^{\mathrm{C}}\right|, \mu_{i}$ is the chemical potential of component $i$ and $\mu_{i}^{\mathrm{C}}$ its value at the critical point ; $\Delta C_{i}^{0}$ is a scale factor and $\beta$ the coexistence curve exponent. Similarly the density difference $\Delta \rho$, the interfacial thickness $L$ and tension $\gamma$ follow the scaling laws :

$$
\begin{aligned}
& \Delta \rho=\Delta \rho^{0} \varepsilon^{\beta} \quad \beta=0.33 \\
& L=L^{0} \varepsilon^{-v} \quad v=2 \beta=0.66 \\
& \gamma=\gamma^{0} \varepsilon^{\varphi} \quad \varphi=4 \beta=1.32 \\
& \beta=0.5 \\
& v=\beta=0.5 \\
& \varphi=3 \beta=1.5
\end{aligned}
$$

$\gamma^{0}, L^{0}, \Delta \rho^{0}$ being scale factors. Unfortunately the present experiments do not give information about the chemical potentials $\mu_{i}$. To avoid this difficulty, we choosed to define $\varepsilon$ by setting the scale factor $\Delta \rho^{0}$ equal to the order of magnitude of $\Delta \rho$ far from the critical points. Here $\Delta \rho^{0}=0.2 \mathrm{~g} / \mathrm{cm}^{3}$ for the two interfaces. Our measurements of $\gamma$ versus $\Delta \rho$ are reported on figure 3 . 


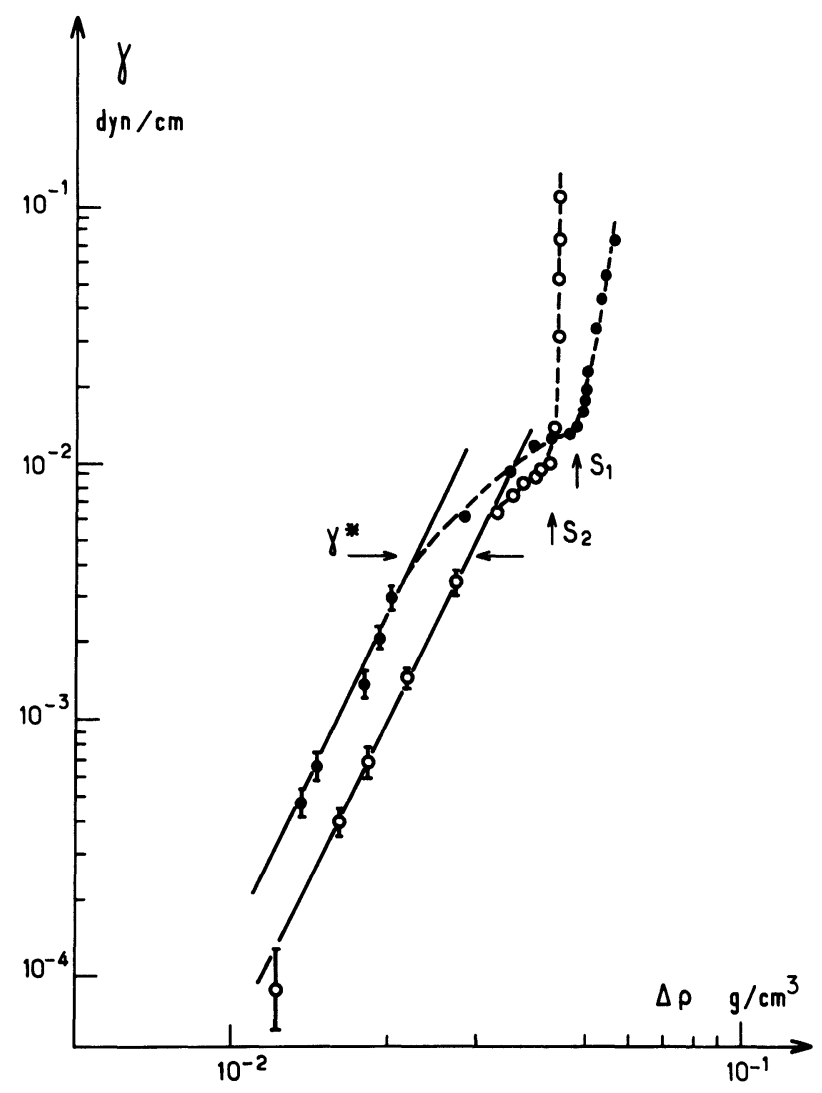

Fig. 3. - Interfacial tensions versus density difference between two phases in equilibrium : $0:$ microemulsion-organic phase $\gamma_{\mathrm{om}}$; $:$ microemulsion-aqueous phase $\gamma_{\mathrm{wm}}$. Vertical arrows indicate the limits between two phases and three phases domain. The horizontal arrows indicate the value $\gamma^{*}$ where $\gamma_{\mathrm{om}}=\gamma_{\mathrm{mw}}=\gamma^{*}$ at the same salinity.

The scaling law is obeyed up to a value $\gamma$ relative to a salinity $S^{*}$ of the three phase domain where the two tensions are equal $\left({ }^{1}\right)$

$$
\gamma_{\mathrm{om}}^{0}=2.6 \times 10^{-2} \mathrm{dyn} . / \mathrm{cm} \text { and } \gamma_{\mathrm{mw}}^{0}=9 \times 10^{-2} \mathrm{dyn} . / \mathrm{cm} .
$$

We find $: \varphi / \beta=4.2 \pm 0.3$ and $4.4 \pm 0.6$ for the two corresponding interfaces (organic phasemicroemulsion and aqueous phase-microemulsion). Above $\gamma^{*}$ the tension between the microemulsion $M$ and the phase $P$ with which it is equilibrium does not change when the microemulsion is replaced with another phase $\mathrm{P}^{\prime}$ also in equilibrium with $\mathrm{P}:$ for $S<S_{1}$, or $S>S_{2}, \mathrm{P}^{\prime}$ is the continuous phase of $\mathrm{M}, S^{*}>S>S_{1} \mathrm{P}^{\prime}$ is the top organic phase, $S^{*}<S<S_{2} \mathrm{P}^{\prime}$ is the bottom aqueous phase. This proves that the structure of the microemulsion phase no longer affects the tension and therefore that $\gamma$ is not related to critical behaviour as evidenced by the deviation to the scaling law. However $\gamma$ values are still low, thus accounting for the low values of the scale factors $\gamma^{0}$. In the three phase domain, where $\Delta \rho^{-\beta}$ plays the role of distance from the critical point, the interfacial thicknesses and the correlation lengths also follow scaling laws : $L^{0}=70 \AA$,

(1) At $S^{*}$ the density differences between upper-middle, and middle-lower phases are not the same. Therefore the two $\gamma$ curves of figure 3 do not intersect at that point. 
$\xi^{0}=4.5 \AA$ and $v / \beta=2 \pm 0.4 . L$ is about 20 times larger than the correlation length $\xi$ in the bulk, although in one or two components systems it has been found of similar amplitude [16].

4. Conclusion. - In conclusion, the behaviour of the microemulsion system in light scattering experiments must be investigated very carefully. When their structure is that of droplets, dilution procedures must be worked out since the interactions are generally noticeably different from hard spheres ones. Sometimes unusual long range interactions seem to appear. We have studied one of such systems and shown that this was in fact related to near critical behaviour. This behaviour can be observed even relatively far from the critical points in multicomponent systems like microemulsions. This explains the origin of part of the ultralow interfacial tensions associated to multiphase behaviour. In the near critical region the experiments are in good agreement with the mode-mode coupling theory. Despite of the large size of the amphiphile molecule, the mean field theory does not apply here, contrary to previously reported observations in simpler amphiphile aqueous solutions [17]. Microemulsion critical behaviour is more marked on interfacial properties $\left(L_{0} \gg \xi_{0}\right)$. The approach to critical points in these systems remains to be understood : the intermediate domain where micelle radius $R$ and correlation lengths $\xi$ are close (case of the boundary $S_{1}$ here) has not yet be satisfactorily described.

\section{References}

[1] Shinoda, K., Friberg, S., Adv. Colloid Interface Sci. 4 (1975) 281.

[2] Ruckenstein, E., ChI, J. C., Faraday Trans. II 71 (1975) 1690.

[3] Miller, C. A., Hwan, R., Benton, W. J., Fort, T., J. Colloid Interface Sci. 61 (1977) 554.

[4] Pouchelon, A., Meunier, J., Langevin, D., Cazabat, A. M., J. Physique Lett. 41 (1980) L-239.

[5] Meunier, J., C. R. Hebd. Séan. Acad. Sci., May 1981, to appear.

[6] See for instance : PUSEY, P. N., in Light scattering in Liquids and macromolecular solutions (Plenum Press) 1980.

[7] Cazabat, A. M., Chatenay, D., Langevin, D., Pouchelon, A., Adv. Colloid Interface Sci., to appear.

[8] SCRIVEN, L. E., Nature (London) 263 (1976) 123.

[9] Lemaire, B., J. Phys. Chem., to appear.

[10] Cazabat, A. M., Chatenay, D., Langevin, D., Pouchelon, A., J. Physique Lett. 41 (1980) L-441.

[11] Swinney, H. L., in Photon correlation and light beating spectroscopy (Plenum Press) 1973.

[12] WidOM, B., in Fundamental problems in statistical mechanics III (North Holland) 1975.

[13] Stanley, H. E., Introduction to phase transitions and critical phenomena (Oxford Univ. Press) 1971.

[14] Griffiths, R. B., Wheeler, J. C., Phys. Rev. A 2 (1970) 1047.

[15] Fleming, P. D., Vinatière, J. E., Glinsman, G. R., J. Phys. Chem. 84 (1980) 1526.

[16] Huang, J. S., Webb, W. W., J. Chem. Phys. 50 (1969) 3677.

[17] Corti, M., Degiorgio, V., Phys. Rev. Lett. 45 (1980) 1045. 